\title{
Criminologie
}

\section{Étude de la récidive post-traitement chez des agresseurs sexuels judiciarisés}

\author{
Bruno Pellerin, Jean Proulx, Marc Ouimet, Yves Paradis, André McKibben et \\ Jocelyn Aubut
}

Volume 29, numéro 1, printemps 1996

Intervenir auprès des délinquants

URI : https://id.erudit.org/iderudit/017382ar

DOI : https://doi.org/10.7202/017382ar

Aller au sommaire du numéro

Éditeur(s)

Les Presses de l'Université de Montréal

ISSN

0316-0041 (imprimé)

1492-1367 (numérique)

Découvrir la revue

Citer cet article

Pellerin, B., Proulx, J., Ouimet, M., Paradis, Y., McKibben, A. \& Aubut, J. (1996).

Étude de la récidive post-traitement chez des agresseurs sexuels judiciarisés.

Criminologie, 29(1), 85-108. https://doi.org/10.7202/017382ar

\section{Résumé de l'article}

The aim of the current study was to assess the role of a treatment program (uncompleted; completed ; extended) on the posttreat-ment recidivism of sexual aggressors. Data on five types of recidivism (sexual ; violent ; property offense ; against a person ; total) were gathered for 102 pedophiles and 70 rapists. The recidivism rates for pedophiles who completed the treatment program was lower than for those of the pedophiles who did not complete the treatment. As to the rapists, the recidivism rates were lower for those who underwent an extended treatment program than for the other two groups. These data show that a treatment program reduces the recidivism rate in sexual aggressors. The optimal duration of treatment, however, varies according to the type of offender. 
ÉTUDE DE LA RÉCIDIVE POST-TRAITEMENT CHEZ DES

AGRESSEURS SEXUELS JUDICIARISÉS

Bruno Pellerin ${ }^{1}$, Jean Proulx ${ }^{12}$,

Marc Ouimet ${ }^{2}$, Yves Paradis ${ }^{1}$,

André McKibben ${ }^{1}$ et Jocelyn Aubut ${ }^{12}$

The aim of the current study was to assess the role of a treatment program (uncompleted; completed; extended) on the posttreatment recidivism of sexual aggressors. Data on five types of recidivism (sexual; violent; property offense; against a person; total) were gathered for 102 pedophiles and 70 rapists. The recidivism rates for pedophiles who completed the treatment program was lower than for those of the pedophiles who did not complete the treatment. As to the rapists, the recidivism rates were lower for those who underwent an extended treatment program than for the other two groups. These data show that a treatment program reduces the recidivism rate in sexual aggressors. The optimal duration of treatment, however, varies according to the type of offender.

Chaque année, un nombre élevé de femmes et d'enfants sont victimes de crimes sexuels. Ainsi, il ne faut pas s'étonner d'entendre des voix s'élever afin de dénoncer les sentences, jugées trop clémentes, imposées aux agresseurs sexuels. Par ailleurs, lorsque récidive un agresseur sexuel ayant reçu un traitement, c'est l'efficacité de l'ensemble des programmes d'intervention destinés à cette clientèle qui est remise en question. En conséquence, la vérification de l'efficacité des programmes de traitement destinés aux agresseurs sexuels constitue un objectif de recherche prioritaire. En effet, si ces programmes s'avèrent efficaces, leur maintien est justifié. Dans le cas contraire, il sera nécessaire d'envisager de nouvelles mesures cliniques et judiciaires.

Plusieurs études furent réalisées afin de vérifier si la participation à un programme de traitement réduit le taux de récidive chez les agresseurs sexuels (Becker et Hunter, 1992; Furby, Weinrott et Blackshaw, 1989; Kelly, 1982; Marshall, Jones, Ward, Johnston et Barbaree, 1991; Rice, Harris et Quinsey, 1993). Toutefois, une proportion importante de ces études ne comporte pas de groupe témoin constitué d'agresseurs sexuels

1. Institut Philippe-Pinel de Montréal, 10905, boul. Henri-Bourassa Est, Montréal (Québec) Canada $\mathrm{H} / \mathrm{C} 1 \mathrm{H} l$

2. Université de Montréal, École de criminologie, Centre international de criminologie comparée, 3150 , rue Jean-Brillant, C. P. 6128, succursale Centre-ville, Montréal (Québec) Canada $\mathrm{H} 3 \mathrm{C} 3 \mathrm{~J}$ 7 
non traités. Ainsi, comme le suggèrent Marshall et coll. (1991), une façon de vérifier l'impact d'un traitement consiste à comparer les taux de récidive des sujets traités d'une étude sans groupe témoin à ceux des sujets non traités d'autres études. Bien que séduisante à première vue, cette approche comporte de nombreuses difficultés. En effet, comme le soulignent plusieurs auteurs (Furby, et coll., 1989; Quinsey, Harris, Rice et Lalumière, 1993; Marques, Day, Nelson et West, 1994), le taux de récidive chez les agresseurs sexuels varie énormément d'une étude à l'autre. Cette variation entre les taux de récidive est fonction de plusieurs facteurs tels la durée de la période de suivi, le type de sujets (violeurs, pédophiles), le niveau de risque, les antécédents judiciaires, le type de milieu thérapeutique et la définition de la récidive. Ainsi, à moins d'apparier les sujets traités et les sujets non traités sur l'ensemble de ces facteurs, il n'est pas possible de vérifier l'impact spécifique d'un traitement. Or, comme le soulignent Quinsey et coll. (1993), un tel appariement est impossible parce que l'information nécessaire est rarement disponible, et parce qu'on ne peut pas contrôler plus de trois variables simultanément.

À notre connaissance, il existe neuf études relatives à l'impact de traitements psychologiques non spécialisés auprès d'agresseurs sexuels et qui comportent un groupe de sujets traités ainsi qu'un groupe témoin constitué de sujets non traités (voir tableau 1). Dans deux de ces études, une procédure d'assignation aléatoire fut utilisée afin de constituer les divers groupes (Peters et Roether, 1971 ; Meyers et Romero, 1980). Aucune de ces deux études ne comporte de différence statistiquement significative quant au taux de récidive sexuelle officielle entre les agresseurs sexuels traités et les non traités. Toutefois, dans ces deux études, il est à noter que les sujets non traités présentent des taux de récidive sexuelle inférieurs à ceux des sujets traités. En ce qui a trait aux sept autres études, le groupe témoin consistait en un échantillon de convenance (absence d'une procédure d'assignation aléatoire) (Davidson, 1984; Dix, 1976; Florida Department of Health and Rehabilitative Services, 1976, 1984; Frisbie, 1969; Hanson, Steffy et Gauthier, 1993; Sturgeon et Taylor, 1980). De nouveau, on observe qu'il n'y a pas de différence statistiquement significative entre le taux de récidive sexuelle officielle des agresseurs sexuels traités et celui des agresseurs sexuels non traités, et ce dans les sept études. Néanmoins, dans le cas de six d'entre elles, les sujets non traités présentent des taux de récidive sexuelle officielle inférieurs à ceux des sujets traités. Ainsi, les programmes de traitement psychologique non spécialisé ne permettent pas de réduire le taux de récidive chez les agresseurs sexuels. Ces résultats ont amené certains auteurs à conclure que ces traitements non spécialisés étaient désuets et que d'autres avenues d'intervention clinique devaient être envisagées (Furby et coll., 1989; Marshall et coll., 1991; Marshall et Pithers, 1994). 
Tableau 1

Études de la récidive sexuelle chez des agresseurs sexuels traités et non traités

\begin{tabular}{|c|c|c|c|c|}
\hline \multicolumn{5}{|c|}{ Traitements psychologiques non spécialisés } \\
\hline Auteurs & Échantillon & $\begin{array}{l}\text { Mesure de } \\
\text { récidive }\end{array}$ & $\begin{array}{l}\text { Période de } \\
\text { suivi }\end{array}$ & $\begin{array}{l}\text { Taux de } \\
\text { récidive } \\
\text { sexuelle }\end{array}$ \\
\hline $\begin{array}{l}\text { Frisbie (1969) } \\
\text { (trait. inteme) } \\
\text { (Atascadero) }\end{array}$ & $\begin{array}{l}617 \text { pédophiles } \\
252 \text { traités }(\mathrm{T} \text { ) } \\
\text { (avec troubles mentaux) } \\
365 \text { non traités (NT) } \\
\text { (sans troubles mentaux) }\end{array}$ & $\begin{array}{l}\text { nouvelle } \\
\text { arrestation pour } \\
\text { crime sexuel }\end{array}$ & $\begin{array}{l}\text { minimum de } \\
3 \text { ans }\end{array}$ & $\begin{array}{l}\mathrm{T}=19,4 \% \\
\mathrm{NT}=11,5 \%\end{array}$ \\
\hline $\begin{array}{l}\text { Peters et Roe- } \\
\text { ther (I971) } \\
\text { (trait. externe) } \\
\text { (J. J. Peters } \\
\text { Inst.) }\end{array}$ & $\begin{array}{l}224 \text { agresseurs } \\
\text { (probation) } \\
\text { (incluant des } \\
\text { homosexuels) } \\
130 \text { traités (T) } \\
94 \text { non traités (NT) } \\
\end{array}$ & \begin{tabular}{|l} 
nouvelle \\
arrestation pour \\
crime sexuel
\end{tabular} & $\begin{array}{l}\text { de } 1 \text { à } 3 \text { ans } \\
\text { (assignation } \\
\text { aléatoire) }\end{array}$ & $\begin{array}{l}\mathrm{T}=7,7 \% \\
\mathrm{NT}-3.2 \%\end{array}$ \\
\hline $\begin{array}{l}\text { Dix (1976) } \\
\text { (trait. inteme) } \\
\text { (Atascadero) }\end{array}$ & $\begin{array}{l}47 \text { agresseurs (violeurs) } \\
\text { (avec troubles mentaux) } \\
24 \text { traités avec succès } \\
\text { (Ta) } \\
6 \text { traités sans succès (Ts) } \\
17 \text { non traités (NT) } \\
\end{array}$ & $\begin{array}{l}\text { nouvelle } \\
\text { arrestation ou } \\
\text { condamnation } \\
\text { pour crime } \\
\text { sexuel }\end{array}$ & $\begin{array}{l}\text { de quelques } \\
\text { mois à } 6,5 \text { ans }\end{array}$ & $\begin{array}{l}\text { Ta }=20,8 \% \\
\text { Ts }=16,6 \% \\
\text { NT }=11,8 \%\end{array}$ \\
\hline $\begin{array}{l}\text { Florida Dept. of } \\
\text { Health (1976) } \\
\text { (trait. interne) }\end{array}$ & $\begin{array}{l}273 \text { agresseurs (en } \\
\text { liberté pendant au moins } \\
12 \text { mois) } \\
39 \text { traités hôpital } 1 \text { (T1) } \\
35 \text { traités hôpital } 2 \text { (T2) } \\
199 \text { non traités (NT) } \\
\end{array}$ & $\begin{array}{l}\text { nouvelle } \\
\text { arrestation pour } \\
\text { crime sexuel }\end{array}$ & 12 mois & $\begin{array}{l}\mathrm{T} 1=5,1 \% \\
\mathrm{~T} 2=5,7 \% \\
\mathrm{NT}=5,0 \%\end{array}$ \\
\hline $\begin{array}{l}\text { Meyers et } \\
\text { Romero (1980) } \\
\text { (trait. externe) } \\
\text { J. J. Peters Inst.) }\end{array}$ & $\begin{array}{l}231 \text { agresseurs } \\
\text { (62\% de violeurs) } \\
147 \text { traités (T) } \\
84 \text { non traités (NT) }\end{array}$ & $\begin{array}{l}\text { nouvelle } \\
\text { arrestation pour } \\
\text { crime sexuel }\end{array}$ & $\begin{array}{l}10 \text { ans } \\
\text { (assignation } \\
\text { aléatoire) }\end{array}$ & $\begin{array}{l}\mathrm{T}=13 \% \\
\mathrm{NT}=7,2 \%\end{array}$ \\
\hline $\begin{array}{l}\text { Sturgeon et } \\
\text { Taylor (1980) } \\
\text { (trait. interne) } \\
\text { (Atascadero) }\end{array}$ & $\begin{array}{l}260 \text { traités }(\mathrm{T}) \\
\text { (avec troubles } \\
\text { mentaux) } \\
122 \text { non traités (NT) } \\
\text { (sans troubles } \\
\text { mentaux) }\end{array}$ & $\begin{array}{l}\text { nouvelle } \\
\text { condamnation } \\
\text { pour crime } \\
\text { sexuel }\end{array}$ & de 1 à 5 ans & $\begin{array}{l}\mathrm{T}=15,4 \% \\
\mathrm{NT}=25 \%\end{array}$ \\
\hline $\begin{array}{l}\text { Davidson } \\
\text { (1984) } \\
\text { (trait. interne) } \\
\text { (4 mois) }\end{array}$ & $\begin{array}{l}n=\text { non spécifié } \\
\text { traités }- \text { pénitencier (T) } \\
\text { non traités = libérés } \\
\text { avant le début du prog. } \\
\text { de traitement (N.T.) }\end{array}$ & $\begin{array}{l}\text { nouvelle } \\
\text { aarestation ou } \\
\text { condamnation } \\
\text { pour crime } \\
\text { sexuel }\end{array}$ & jusqu'a 5 ans & $\begin{array}{l}\text { récidive sex. = } \\
\text { non spécifiée } \\
\text { mais selon } \\
\text { Quinsey et coll. } \\
\text { (1993) } \\
\mathrm{T}= \\
\text { pourcentage de } \\
\text { récidive sex. } \\
\text { (arrestation) } \\
\text { signif. supérieur } \\
\text { à celui des NT }\end{array}$ \\
\hline
\end{tabular}




\begin{tabular}{|c|c|c|c|c|}
\hline \multicolumn{5}{|c|}{ Traitements psychologiques non spécialisés } \\
\hline Auteurs & Échantillon & $\begin{array}{l}\text { Mesure de } \\
\text { récidive }\end{array}$ & $\begin{array}{l}\text { Période de } \\
\text { suivi }\end{array}$ & $\begin{array}{l}\text { Taux de } \\
\text { récidive } \\
\text { sexuelle }\end{array}$ \\
\hline $\begin{array}{l}\text { Florida Dept. of } \\
\text { Health (1984) } \\
\text { (trait. interne) }\end{array}$ & $\begin{array}{l}59 \text { traités (T) } \\
31 \text { trait. non compl. (Tnc) } \\
20 \text { en attente de tratt. (Tat) } \\
92 \text { non traités (NT) }\end{array}$ & $\begin{array}{l}\text { nouvelle } \\
\text { arrestation pour } \\
\text { crime sexuel }\end{array}$ & $\begin{array}{l}\text { de } 2 \text { à } 84 \text { mois } \\
\text { (moyenne - } \\
30 \text { mois) }\end{array}$ & $\begin{array}{l}\mathrm{T}=13,6 \% \\
\mathrm{Tnc}=6,5 \% \\
\mathrm{Tat}=5 \% \\
\mathrm{NT}=10,9 \%\end{array}$ \\
\hline $\begin{array}{l}\text { Hanson, Steffy } \\
\text { et Gauthier } \\
\text { (1993) } \\
\text { (trait. interne) } \\
\text { (durée de } \\
5 \text { mois) }\end{array}$ & $\begin{array}{l}106 \text { pédophiles traités }(\mathrm{T}) \\
31 \text { pédophiles non } \\
\text { traités (NT1) } \\
\text { (lib. avant début prog. } \\
\text { trait.) } \\
60 \text { pédophiles non } \\
\text { traités (NT2) } \\
\text { (durant même période } \\
\text { que T) }\end{array}$ & $\begin{array}{l}\text { nouvelle } \\
\text { condamnation } \\
\text { pour crime } \\
\text { sexuel (incluant } \\
\text { crimes violents) }\end{array}$ & $\begin{array}{l}\text { jusqu'à } 31 \text { ans } \\
\text { (moyenne } \\
\mathrm{T}=19 \text { ans) } \\
\text { (moyenne } \\
\text { NT1 = } 28 \text { ans) } \\
\text { (moyenne } \\
\text { NT2 = } 20 \text { ans) }\end{array}$ & $\begin{array}{l}\mathrm{T}=44 \% \\
\mathrm{NT} 1=48 \% \\
\mathrm{NT} 2=33 \%\end{array}$ \\
\hline \multicolumn{5}{|c|}{ Traitements cognitivo-comportementaux } \\
\hline $\begin{array}{l}\text { Marshall et Bar. } \\
\text { baree (1988) } \\
\text { (trait. externe } \\
\text { multimodal) } \\
\text { (durée de } \\
4 \text { mois) }\end{array}$ & $\begin{array}{l}68 \text { pédophiles traités (T) } \\
58 \text { pédophiles non } \\
\text { traités (NT) } \\
\text { (incarc./habite trop loin) }\end{array}$ & $\begin{array}{l}\text { avoir commis } \\
\text { nouveau crime } \\
\text { sexuel (selon } \\
\text { les dossiers de } \\
\text { la DPJ) } \\
\text { (récidive non } \\
\text { officielle) }\end{array}$ & $\begin{array}{l}\text { de } 1 \text { à } 11 \text { ans } \\
\text { (moyenne = } \\
4 \text { ans) }\end{array}$ & $\begin{array}{l}T=13,2 \% \\
\text { NT }=34,5 \%\end{array}$ \\
\hline $\begin{array}{l}\text { Rice, Quinsey } \\
\text { et Harris (1991) } \\
\text { (trait. interne) }\end{array}$ & $\begin{array}{l}50 \text { pédophiles traités (T) } \\
\text { (avec troubles mentaux) } \\
86 \text { pédophiles non } \\
\text { Iraités (NT) } \\
\text { (statut pré-sentenciel) } \\
\text { (sans troubles mentaux) }\end{array}$ & $\begin{array}{l}\text { nouvelle } \\
\text { condamnation } \\
\text { pour crime } \\
\text { sexuel }\end{array}$ & $\begin{array}{l}6,3 \text { ans en } \\
\text { moyenne }\end{array}$ & $\begin{array}{l}T(n=29)= \\
38 \% \\
N T(n=29)= \\
31 \%\end{array}$ \\
\hline $\begin{array}{l}\text { Marques, Day, } \\
\text { Nelson et West } \\
(1994) \\
\text { (trait. int. + } \\
\text { ext.) } \\
\text { (durée de } 3 \text { ans) }\end{array}$ & $\begin{array}{l}233 \text { pédophiles }(P) \\
58 \text { violeurs }(\mathrm{V}) \\
98 \text { traités(T) } \\
(\mathrm{P}=76 ; \mathrm{V}=22) \\
(\mathrm{I} \text { an de trait. complété) } \\
97 \text { non traités mais } \\
\text { motıvés à être traités } \\
(\mathrm{NTM})(\mathrm{P}=79 ; \mathrm{V}-18) \\
96 \text { non traités ayant } \\
\text { refusé d'être traités } \\
\text { (NTR) }(\mathrm{P}=78 ; \mathrm{V}=18) \\
8 \text { traités non complétés } \\
(\mathrm{TNC})(\mathrm{P}=7 ; \mathrm{V}=1)\end{array}$ & $\begin{array}{l}\text { nouvelle } \\
\text { arrestation pour } \\
\text { crime sexuel }\end{array}$ & 5 ans & $\begin{array}{l}(P+V) T=8,2 \% \\
\text { NTM }=13,4 \% \\
\text { NTR }=12,5 \% \\
(P) T=7,9 \% \\
\text { NTM }=10,1 \% \\
\text { NTR }=12,8 \% \\
(V) T=9.1 \% \\
\text { NTM }=27,8 \% \\
\text { NTR }=11,1 \% \\
(P+T)=8,2 \% \\
T N C=37,5 \%\end{array}$ \\
\hline
\end{tabular}


Dans la documentation scientifique récente, on note deux études qui portent sur l'impact de traitements cognitivo-comportementaux auprès d'agresseurs sexuels qui comportent un groupe de sujets traités et un groupe témoin de convenance constitué de sujets non traités (Marshall et Barbaree, 1988; Rice, Quinsey et Harris, 1991) (voir tableau 1). En ce qui a trait à l'étude de Marshall et Barbaree, le taux de récidive sexuelle non officielle de pédophiles traités $(13,2 \%)$ est significativement inférieur à celui de pédophiles non traités $(34,5 \%)$. Cependant, il n'y a pas de différence statistiquement significative entre le taux de récidive sexuelle officielle des pédophiles traités (hétérosexuels : 7,5\%; homosexuels : 5,5\%; incestueux : $2,9 \%$ ) et celui des pédophiles non traités (hétérosexuels: 19,2\%; homosexuels : 17,9\%; incestueux : 7,0\%). Quant aux résultats de l'étude de Rice et coll. (1991), ils indiquent que les pédophiles traités (38\%) présentent un taux de récidive sexuelle ou violente qui ne diffère pas de manière statistiquement significative de celui des pédophiles non traités (31\%). Cependant, il importe de souligner que les deux groupes de cette étude n'étaient pas comparables, puisque les sujets traités présentaient des troubles mentaux, ce qui n'était pas le cas des sujets non traités. Ainsi, sur la base de ces deux études, il est difficile de conclure à l'efficacité des programmes de traitement cognitivo-comportementaux destinés aux agresseurs sexuels.

Parmi les études portant sur l'efficacité des programmes de traitement cognitivo-comportementaux, l'étude de Marques et coll. (1994) est la seule dans laquelle une procédure d'assignation aléatoire fut utilisée pour la constitution des groupes d'agresseurs sexuels. Cette étude comporte un groupe d'agresseurs sexuels traités (violeurs et pédophiles) (T), un groupe d'agresseurs sexuels non traités et ayant manifesté le désir de l'être (NTM) et, finalement, un groupe d'agresseurs sexuels ayant refusé le traitement (NTR). La seule différence statistiquement significative observée dans cette étude est un taux de récidive violente plus bas chez les pédophiles traités $(4,0 \%)$ en comparaison des pédophiles non traités et ayant manifesté le désir de l'être $(13,9 \%)$. Toutefois, il est à noter que le taux de récidive violente des pédophiles traités $(4,0 \%)$ est plus bas que celui des pédophiles ayant refusé de l'être $(6,4 \%)$. De plus, le taux de récidive sexuelle des pédophiles traités $(7,9 \%)$ est inférieur à ceux des deux groupes de pédophiles non traités (NTM : 10,1\%; NTR : $12,8 \%$ ). Dans le cas des violeurs, il n'y a aucune différence statistiquement significative entre le taux de récidive sexuelle $(9,1 \%)$ des violeurs traités et ceux des deux groupes de violeurs non traités (NTM : $27,8 \%$; NTR : $11,1 \%$ ). De plus, il n'y a pas de différence statistiquement significative entre le taux de récidive violente des violeurs traités $(22,7 \%)$ et ceux des deux groupes de violeurs non traités (NTM : $33,3 \%$; NTR : $22,2 \%$ ). Malgré la rigueur scientifique manifestêe dans la constitution des groupes, les résultats de cette étude ne 
permettent pas de conclure sans équivoque que les programmes de traitement cognitivo-comportementaux réduisent les taux de récidive chez les violeurs et chez les pédophiles.

Les résultats de l'étude de Marques et coll. (1994) ont également permis de mettre au jour l'importance de considérer le taux de récidive des sujets n'ayant pas complété le traitement. Pour reprendre leurs termes, treatment program value is measured not only by the success of those who complete it but also by the number who refuse the interventions or dropout after beginning treatment (p. 33). En effet, parmi les 106 sujets traités de leur étude, i] y en a 8 (7 pédophiles, 1 violeur) qui n'ont pas complété le traitement (traitement d'une durée inférieure à 12 mois) et il y en a 98 (76 pédophiles, 22 violeurs) qui ont complété le traitement (traitement d'une durée égale ou supérieure à 12 mois). Ainsi, le taux de récidive sexuelle des agresseurs sexuels (violeurs, pédophiles) n'ayant pas complété le traitement $(37,5 \%)$ s'avère significativement plus élevé que celui de ceux qui l'ont complété $(8,2 \%)$. De plus, le taux de récidive violente des agresseurs sexuels n'ayant pas complété le traitement $(37,5 \%)$ est plus élevé que celui de ceux l'ayant complété $(8,2 \%)$. En conséquence, les résultats de cette étude démontrent que l'abandon précoce d'un programme de traitement est un facteur qui influe sur les taux de récidive chez des agresseurs sexuels. Ces résultats donnent du poids à l'opinion de Quinsey et coll. (1993) selon laquelle in the absence of data about the numbers and outcomes of treatment refusers, dropouts, and noncompliers, data about the outcome of those who complete treatment, no matter how positive (or negative), cannot provide evidence of treatment efficacity (p. 515).

Ainsi, l'étude de Marques et coll. (1994) est la seule dans laquelle les taux de récidive d'agresseurs sexuels n'ayant pas complété un programme de traitement furent comparés à ceux d'agresseurs en ayant complété un. Malheureusement, à cause du nombre restreint de sujets n'ayant pas complété le traitement, Marques et coll. n'ont pas effectué d'analyses statistiques séparées pour les violeurs $(\mathrm{N}=1)$ et les pédophiles $(\mathrm{N}=7)$. En conséquence, le but de la présente étude est de comparer les taux de récidive de violeurs et de pédophiles n'ayant pas complété un programme de traitement avec ceux de violeurs et de pédophiles en ayant complété un.

\section{MÉTHODOLOGIE}

\section{Sujets}

Entre 1978 et 1991, 382 hommes ayant des antécédents judiciaires officiels relatifs à au moins un délit sexuel furent évalués à l'Institut Philippe-Pinel de Montréal, hôpital psychiatrique sécuritaire, ou à la 
clinique externe qui lui est rattachée. Parmi ces sujets, 172 reçurent un traitement dont le statut est fonction de sa durée. Ainsi, les sujets ayant reçu un traitement dont la durée est inférieure à 12 mois sont classés dans la catégorie «non-complété » $(\mathrm{N}=63)$, ceux pour lesquels il fut d'une durée se situant entre 12 et 24 mois se retrouvent dans la catégorie "complété " $(\mathrm{N}=55)$ et ceux qui reçurent plus de 24 mois de traitement sont classés dans la catégorie "prolongé » $(\mathrm{N}=54)$. Cette catégorisation est fonction de la durée théorique du traitement, soit 24 mois, ainsi que d'un souci de constituer des groupes comparables à ceux de l'étude de Marques et coll. (1994).

Sur la base des informations contenues dans le rapport d'enquête policière (déclaration des victimes et des témoins) ainsi que de celles se trouvant dans le rapport d'évaluation clinique, les sujets traités furent classés dans la catégorie "violeur» $(\mathrm{N}=70)$ ou dans la catégorie "pédophile" $(N=102)$. Ainsi, afin d'être classé dans la catégorie «violeur", un sujet doit être l'auteur d'au moins une agression sexuelle contre une femme adulte ou contre une adolescente pubère, alors que pour être classé dans la catégorie «pédophile», un sujet doit avoir commis au moins une agression sexuelle contre un enfant prépubère (de sexe masculin ou féminin) ou contre un adolescent pubère.

Trente-huit sujets furent exclus de la présente étude parce que les rapports sur leurs antécédents judiciaires officiels ne spécifiaient que des actes de nuisance sexuelle, soit de l'exhibitionnisme ou du voyeurisme. De même, 248 sujets furent exclus parce qu'ils ne présentaient pas d'antécédents judiciaires sexuels officiels, et ce même s'ils furent évalués ou traités pour une problématique reliée à l'agression sexuelle. Finalement, 34 sujets étaient toujours incarcérés au moment de la fin de la période de suivi. En résumé, de 1978 à 1991, 702 sujets furent évalués ou traités à l'Institut Philippe-Pinel de Montréal pour une problématique de déviance sexuelle. Ainsi, dans le cas de la présente étude, seuls les 172 sujets ayant reçu un traitement et présentant des antécédents judiciaires officiels relatifs à une agression sexuelle (viol, pédophilie) ont été retenus.

\section{Traitement}

Afin d'être sélectionné en vue d'une participation au programme de traitement (interne ou externe) pour agresseurs sexuels, un sujet devait répondre aux critères suivants: 1) ne pas présenter de retard intellectuel; 2) ne pas souffrir d'un trouble mental grave (ex.: schizophrénie, trouble de l'humeur); 3) reconnaître qu'il est l'auteur du délit pour lequel il est judiciarisé et actuellement en traitement; 4) reconnaître le caractère délictuel, déviant, des actes posés; 5) manifester le désir de s'impliquer dans un programme de traitement. 
Tout d'abord, en ce qui concerne le programme de traitement à l'interne, il fut institué en 1978. Toutefois, il importe de souligner que ce programme a évolué au cours des ans. Ainsi, en 1991, les principales composantes de ce programme de traitement étaient les suivantes : 1) atelier de prévention de la récidive; 2 ) atelier d'entraînement aux habiletés sociales ; 3) atelier d'entraînement à la gestion du stress ; 4) atelier d'entraînement à la maîtrise de la colère; 5) cours d'éducation sexuelle; 6) atelier d'entraînement à la gestion de la consommation d'alcool et de drogue; 7) techniques comportementales visant à modifier les préférences sexuelles; 8) séances de thérapie familiale et conjugale; 9) castration chimique. Il est à spécifier que le programme de traitement est adapté en fonction des difficultés propres à chaque agresseur sexuel. Ainsi, les quatre dernières composantes du programme de traitement, mentionnées ci-haut, sont facultatives.

De plus, chaque agresseur sexuel en traitement reçoit un accompagnement individuel. Finalement, l'ensemble des activités à l'unité de traitement (ex.: sports, tâches ménagères) favorisent la généralisation des habiletés apprises lors des divers ateliers. Pour obtenir une description plus détaillée du programme de traitement interne pour agresseurs sexuels de l'Institut Philippe-Pinel de Montréal, la consultation d'autres documents s'avère nécessaire (Aubut, 1993; McKibben et Proulx, 1991).

En 1986, un programme de traitement externe pour agresseurs sexuels fut instauré. Ce programme comporte plusieurs des composantes du traitement à l'interne, soit : 1) un atelier de prévention de la récidive; 2) un atelier d'entraînement aux habiletés sociales; 3 ) un cours d'éducation sexuelle; 4) des séances de thérapie familiale et conjugale ; 5) la castration chimique. En outre, il comporte des suivis individuels et de groupe qui se situent dans une perspective psychodynamique.

\section{Évaluation phallométrique}

Cette méthode d'évaluation consiste à enregistrer les réponses péniennes d'un sujet à l'aide d'un extensomètre au mercure (Bancroft, Jones et Pullan, 1966) lors de la présentation de stimuli sexuels déviants et non déviants (Proulx, 1989). Le laboratoire d'évaluation psychosexuelle comporte deux pièces. La première contient l'appareil d'enregistrement des données, soit le polygraphe, ainsi que l'appareil pour présenter les stimuli, c'est-à-dire le lecteur de bandes sonores. L'évaluateur est assis dans cette pièce et il effectue la présentation des stimuli ainsi que l'enregistrement des données. Dans une pièce adjacente, le sujet est installé sur une chaise. On trouve dans cette seconde pièce les écouteurs dont le sujet a besoin pour les bandes sonores. 
Les stimuli utilisés pour évaluer les violeurs sont des traductions françaises des enregistrements en anglais produits par Abel, Blanchard, Becker et Djenderedjian (1978). La validité discriminante des traductions françaises de ces stimuli fut démontrée au cours de deux études (Earls et Proulx, 1986; Proulx, Aubut, McKibben et Côté, 1994). Les catégories de stimuli sont les suivantes: 1) relation sexuelle avec une femme adulte consentante; 2) viol avec humiliation d'une femme adulte; 3 ) viol avec violence physique d'une femme adulte; 4) agression physique non sexuelle d'une femme adulte; 5) stimulus neutre (ex.: les avantages de porter la ceinture de sécurité dans une automobile). Lors des évaluations, deux bandes sonores pour chacune de ces cinq catégories de stimuli furent présentées à chaque sujet. La durée moyenne de ces stimuli est de 208,57 $\mathrm{s}$. (e.t. : 29,82).

Quant aux stimuli présentés aux pédophiles, ils consistent en đes traductions françaises d'enregistrements en anglais produits par Quinsey et Chaplin (1988). La validité discriminante de ces stimuli fut vérifiée au cours de deux études (De Gagné, 1987; Proulx et coll., 1995). Les catégories de stimuli sont les suivantes: 1) relation sexuelle avec une personne adulte consentante ; 2 ) attouchement sur un enfant de huit ans non résistant; 3) attouchement sur un enfant de huit ans résistant; 4) pénétration génitale ou anale d'un enfant de huit ans résistant; 5) agression physique non sexuelle d'un enfant de huit ans résistant. Lors de l'évaluation phallométrique d'un pédophile, une série de cinq stimuli à contenu homosexuel, une autre série à contenu hétérosexuel et un stimulus neutre sont présentés au sujet. La durée moyenne de ces stimuli est de 121,8 ] s. (e.t. : 12,89).

L'évaluation phallométrique fut introduite à l'Institut Philippe-Pinel de Montréal en 1984. En conséquence, pour les sujets évalués et traités antérieurement, il n'existe pas de données phallométriques. Par ailleurs, les données phallométriques de certains sujets traités ne furent pas retenues parce que l'amplitude maximale d'érection enregistrée n'était pas supérieure à $1 \mathrm{~mm}$ de diamètre pénien. Cette amplitude minimale représente approximativement de $10 \%$ à $15 \%$ d'une érection complète. Or, une telle amplitude d'érection est insuffisante pour être interprétable (Quinsey et Laws, 1990). Ainsi, il n'existe des données phallométriques pré-traitement analysables que pour 37 violeurs et 59 pédophiles, soit pour $55,8 \%$ des agresseurs sexuels traités lors de cette étude. Quant aux données phallométriques post-traitement, elles n'existent et ne sont utilisables que pour 18 violeurs et 26 pédophiles traités, soit pour $25,6 \%$ des agresseurs sexuels traités lors de cette étude.

Pour chaque agresseur sexuel traité dont les données phallométriques étaient analysables, des indices de déviance furent calculés. Un indice de déviance consiste en un rapport où le numérateur est constitué de la réponse 
pénienne maximale obtenue lors des stimuli déviants et le dénominateur correspond à celle présentée lors des stimuli non déviants. Ainsi, pour chaque violeur, trois indices de déviance furent calculés, soit : 1) l'indice de viol physique; 2) l'indice de viol-humiliation; 3) l'indice d'agression physique non sexuelle. Dans le cas de chaque pédophile, sept indices de déviance furent calculés, soit: 1) l'indice de pédophilie homosexuelle attouchement, non résistant; 2) l'indice de pédophilie homosexuelle attouchement, résistant ; 3) l'indice de pédophilie homosexuelle - pénétration, résistant ; 4) l'indice de pédophilie hétérosexuelle - attouchement, non résistant; 5) l'indice de pédophilie hétérosexuelle - attouchement, résistant; 6) l'indice de pédophilie hétérosexuelle - pénétration, résistant; 7) l'indice de pédophilie globale.

\section{Évaluation psychométrique}

À l'Institut Philippe-Pinel de Montréal, l'utilisation systématique d'instruments psychométriques pour évaluer les agresseurs sexuels a débuté en 1987. Ces instruments furent introduits en plusieurs étapes. Ainsi, les données psychométriques prétraitement ne sont disponibles, dans le meilleur des cas, que pour 33 pédophiles et 20 violeurs. Les instruments psychométriques en usage en 1991 étaient les suivants : l'inventaire d'anxiété situationnelle et de trait (Spielberger, Gorsuch et Lushene, 1970), l'inventaire de dépression de Beck (Beck, Ward, Mendelson, Mock et Erbaugh, 1961), l'inventaire de propension à la colère (Novaco, 1975), l'échelle d'intimité sociale de Miller (Miller et Lefcourt, 1982), l'échelle de satisfaction de vie (Pavot, 1993), l'échelle de croyances sur le viol (Burt, 1980), l'échelle de croyances pédophiles (Abel et coll., 1989), l'inventaire clinique multiaxial de Millon (MCMI) (Millon, 1983).

\section{Antécédents judiciaires et caractéristiques du dernier délít}

Pour chaque sujet, des informations relatives à ses antécédents judiciaires antérieurs à son traitement à l'Institut Philippe-Pinel de Montréal furent obtenues à partir des fichiers de la Gendarmerie royale du Canada. Les informations retenues sont les suivantes : 1) nombre de chefs d'accusa tion pour des crimes sexuels; 2) nombre de chefs d'accusation pour des crimes violents non sexuels; 3) nombre de chefs d'accusation pour des crimes non sexuels et non violents; 4) nombre de sentences; 5) durée de la dernière sentence; 6) nombre de victimes en relation avec la dernière sentence; 7) âge de la dernière victime officielle; 8) sexe de la dernière victime officielle; 9) lien entre l'agresseur et la dernière victime officielle.

\section{Données démographiques}

Cette section concerne l'âge de l'agresseur ainsi que son statut civil. Les sujets qui étaient célibataires, divorcés ou séparés au moment du 
dernier délit furent classés dans la catégorie « vit seul », alors que ceux qui étaient mariés ou qui vivaient en concubinage au moment du dernier délit furent classés dans la catégorie «ne vit pas seul ».

\section{Données sur la récidive}

Pour chaque sujet, des données relatives à ses activités criminelles judiciarisées postérieures à son traitement à l'Institut Philippe-Pinel de Montréal furent obtenues à partir des fichiers du service d'identité judiciaire de la Gendarmerie royale du Canada. Ainsi, ne furent retenues que les données sur la récidive officielle (une nouvelle condamnation). Ces données sont conservatrices, mais elles constituent une mesure plus objective de la récidive que les données non officielles obtenues auprès d'autres sources d'information (Quinsey et coll., 1993).

Pour tous les sujets, Ja période de suivi post-traitement se termine en 1994, mais elle débute à différents moments se situant entre 1978 et 1993 (moyenne $=55,9$ mois; étendue : de 1 à 155 mois) (voir tableau 2). Pour les sujets ayant participé au programme de traitement en institution, le début de la période de suivi correspond au jour où se sont écoulés les deux tiers de la dernière sentence. Cet estimé correspond au moment habituel de remise en liberté dans le cas des crimes sexuels. Pour les agresseurs sexuels ayant participé au programme de traitement en externe, la période de suivi débute au moment où s'arrête leur participation à ce programme.

Tableau 2

Moyenne (écart type) de période de suivi post-traitement (nombre de mois) chez les agresseurs sexuels traités entre 1978 et 1991

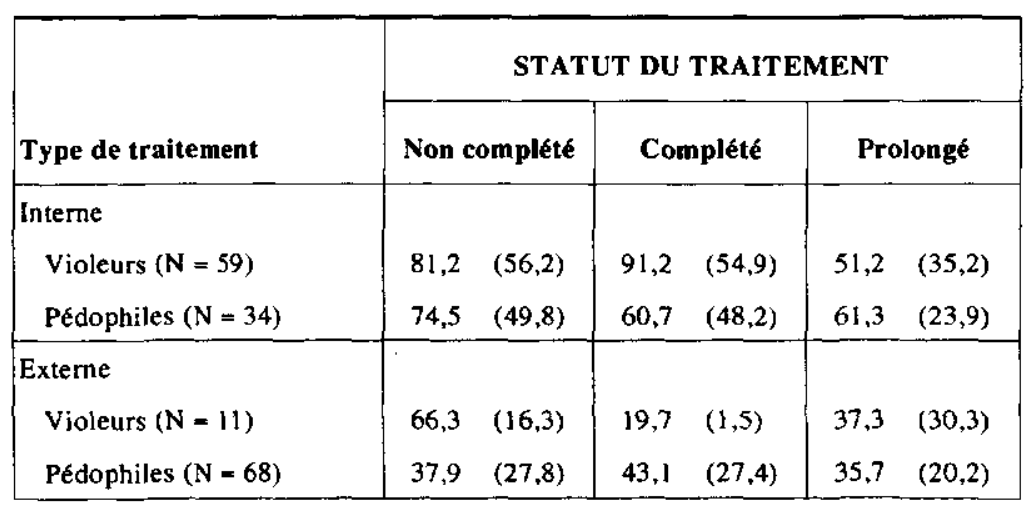

La récidive fut définie de cinq façons: 1) la récidive sexuelle; 2) la récidive violente ; 3 ) la récidive contre les biens; 4) la récidive contre les personnes; 5) la récidive totale. Tous ces cas de récidive renvoient à des condamnations pour des délits perpétrés durant la période de suivi. La 
récidive sexuelle consiste en une nouvelle condamnation pour délit sexuel. La récidive violente consiste en une nouvelle condamnation pour délit violent et non sexuel. La récidive contre les biens consiste en une nouvelle condamnation pour crime contre les biens ne comportant pas d'éléments sexuels ni d'éléments violents. La récidive contre les personnes consiste en une condamnation pour un délit sexuel ou violent. Finalement, la récidive totale consiste en une condamnation pour tout type de crime.

\section{RÉSULTATS}

\section{Pédophiles}

Au tableau 3, on trouve les taux de récidive (sexuelle, violente, contre les biens, contre les personnes, totale) des pédophiles dont le traitement est soit non complété, soit complété, soit prolongé. Puisqu'il n'y a pas de lien significatif entre le type de milieu thérapeutique (interne et externe) et le statut du traitement (non complété, complété, prolongé), seules les analyses portant sur les données des deux types de traitement regroupés seront présentées. Ainsi, il n'y a pas de lien significatif entre le statut du traitement et les taux de récidive sexuelle, $\chi^{2}(2)=4,0, p=, 1$, phi $=, 20$, et violente, $\chi^{2}(2)$ $=5,0, p=, 08$, phi $=, 22$, et contre les personnes, $\chi^{2}(2)=5,9, p=, 05$, phi $=, 24$. Cependant, il y a un lien significatif entre le statut du traitement et les taux de récidive contre les biens, $\chi^{2}(2)=8,4, p=02$, phi $=, 29$, et totale, $\chi^{2}(2)=9,4, p=, 001$, phi $=, 30$.

\section{Tableau 3}

Taux (fréquence) de récidive chez des pédophiles selon le statut du traitement

\begin{tabular}{|l|c|c|r|r|r|r|}
\hline \multicolumn{2}{|l|}{} & \multicolumn{6}{|c|}{ TYPE DE RÉCIDIVE } \\
\hline Traitement & $\mathrm{N}$ & Sexuelle & Violente & $\begin{array}{l}\text { Contre les } \\
\text { biens }\end{array}$ & $\begin{array}{l}\text { Contre les } \\
\text { personnes }\end{array}$ & Totale \\
\hline $\begin{array}{l}\text { Non complété } \\
\text { (moins de 12 mois) }\end{array}$ & 39 & $20,5 \%(8)$ & $7,7 \%(3)$ & $25,6 \%(10)$ & $20,5 \%(8)$ & $33,3 \%(13)$ \\
\hline $\begin{array}{l}\text { Complété } \\
\text { (de 12 à 24 mois) }\end{array}$ & 35 & $5,7 \%(2)$ & $0 \%(0)$ & $2,9 \%(1)$ & $5,7 \%(2)$ & $5,7 \%(2)$ \\
\hline $\begin{array}{l}\text { Prolongé } \\
\text { (plus de 24 mois) }\end{array}$ & 28 & $21,4 \%(6)$ & $14,3 \%(4)$ & $10,7 \%(3)$ & $28,6 \%(8)$ & $32,1 \%(9)$ \\
\hline
\end{tabular}

Des analyses de régression logistique furent également effectuées afin de vérifier s'il existe des liens significatifs entre les taux de récidive et le statut du traitement chez les pédophiles. Lors de ces analyses, il fut contrôlé pour l'impact de variables qui affectent les taux de récidive : l'âge du sujet, ses antécédents judiciaires sexuels et contre les biens, le sexe de la dernière 
victime, la durée de la période de suivj et, finalement, le type de milieu thérapeutique (Proulx et coll., 1995). Toutefois, nous n'avons pas contrôlé les variables dynamiques, soit les préférences sexuelles et les caractéristiques de la personnalité, parce que le nombre de sujets pour lesquels ces données existaient était insuffisant. Ainsi, lors d'une première étape, les variables qui exercent un effet sur les taux de récidive furent introduites dans les analyses de régression logistique. Puis, lors d'une seconde étape, la variable "statut du traitement" fut introduite dans les analyses de régression logistique. La différence entre la statistique du chi-carré décrivant l'ajustement du modèle dans la première étape et celle obtenue lors de la seconde, soit l'amélioration de l'ajustement (improvement), permet de vérifier si l'introduction de la variable «statut du traitement » dans l'équation de régression améliore le modèle de prédiction de la probabilité de récidive en comparaison de celui établi sans eux. La nature catégorielle de la variable «statut du traitement» a conduit à la création de vecteurs dichotomiques qui permettent d'utiliser la catégorie «traitement complété » comme la catégorie de référence à laquelle sont comparées les catégories " traitement non complété » et «traitement prolongé » lors de la seconde étape des analyses de régression logistique.

Au tableau 4, on trouve, dans le cas de cinq types de récidive chez des pédophiles traités, des statistiques décrivant la performance des modèles soumis aux équations de régression logistique soit : l'amélioration du chicarré, le nombre de degrés de liberté, le niveau de signification du $\chi^{2}$. Des statistiques pour chacune des deux étapes de ces analyses hiérarchiques sont présentées. En ce qui a trait à la récidive sexuelle, l'amélioration du chi-carré pour la variable statut du traitement est marginalement significative $(p=05)$. Toutefois, elle est significative pour les quatre autres formes de récidive. Dans le cas de la récidive violente, malgré une amélioration significative du chi-carré, le fait de compléter le traitement n'affecte pas de manière notable la probabilité de récidive en comparaison des deux autres catégories de statut du traitement. En ce qui concerne la récidive contre les biens, les sujets qui ont complété le traitement présentent une probabilité de récidive plus faible que ceux qui n'ont pas complété le traitement, $\mathbf{R}$ (coefficient de corrélation partielle) $=, 24$, OR (odds ratio, rapport de cotes) $=$ 59,58 , Wald $=4,48, p<, 05$. En ce qui a trait à la récidive contre les personnes, les sujets qui ont complété le traitement présentent une probabilité de récidive plus faible que les sujets ayant reçu un traitement prolongé, $\mathrm{R}=, 27, \mathrm{OR}=18,3$, Wald $=7,37, p<, 01$. Finalement, en ce qui concerne la récidive totale, les sujets qui ont complété le traitement présentent une probabilité de récidive plus faible que les sujets qui ne l'ont pas complété, $\mathbf{R}=19, \mathrm{OR}=12,17$, Wald $=4,71, p<, 05$, ainsi que celui des sujets qui ont reçu un traitement prolongé $R=, 29, \mathrm{OR}=30,93$, Wald $=$ $8,52, p<, 01$. 


\section{Tableau 4}

Statistiques relatives aux analyses de régression logistique pour cinq types de récidive chez des pédophiles traités $(\mathrm{N}=102)$

\begin{tabular}{|c|c|c|c|c|}
\hline Type de récidive & $\begin{array}{c}\text { Étape de } \\
\text { l'analyse de } \\
\text { régression } \\
\text { logistique }\end{array}$ & $\chi^{2}$ & DL & $p$ \\
\hline Sexuelle & $\begin{array}{l}\text { Étape } 1 \\
\text { Étape } 2\end{array}$ & $\begin{array}{r}21,75 \\
5,93\end{array}$ & $\begin{array}{l}6 \\
2\end{array}$ & $\begin{array}{l}.001 \\
.05\end{array}$ \\
\hline Violente & $\begin{array}{l}\text { Étape } 1 \\
\text { Étape } 2\end{array}$ & $\begin{array}{r}6,56 \\
10,81\end{array}$ & $\begin{array}{l}6 \\
2\end{array}$ & $\begin{array}{l}.36 \\
.005\end{array}$ \\
\hline Contre les biens & $\begin{array}{l}\text { Étape } 1 \\
\text { Étape } 2\end{array}$ & $\begin{array}{r}31,48 \\
7,76\end{array}$ & $\begin{array}{l}6 \\
2\end{array}$ & $\begin{array}{l}, 001 \\
, 02\end{array}$ \\
\hline Contre les personnes & $\begin{array}{l}\text { Étape } 1 \\
\text { Étape } 2\end{array}$ & $\begin{array}{l}19,41 \\
10,81\end{array}$ & $\begin{array}{l}6 \\
2\end{array}$ & $\begin{array}{l}.004 \\
, 005\end{array}$ \\
\hline Totale & $\begin{array}{l}\text { Étape } 1 \\
\text { Étape } 2\end{array}$ & $\begin{array}{l}29,10 \\
12,93\end{array}$ & $\begin{array}{l}6 \\
2\end{array}$ & $\begin{array}{l}.001 \\
.002\end{array}$ \\
\hline \multicolumn{5}{|c|}{$\begin{array}{l}\text { Nombre de sujets }(\mathrm{N}) \text {. } \\
\text { Amélioration du chi-carré }\left(\chi^{2}\right) \text {. } \\
\text { Nombre de degré de liberté (DL). } \\
\text { Signification }(p) \text {. } \\
\text { Étape } 1: \text { Statistiques de l'équation avec les variables contrôles qui exercent un } \\
\text { effet sur le taux de récidive. } \\
\text { Étape } 2 \text { : Statistiques de l'équation avec la variable traitement, après soustraction } \\
\text { de l'effet des variables contrôles (Improvement: amélioration de l'ajustement). }\end{array}$} \\
\hline
\end{tabular}

Au tableau 5, on trouve des statistiques relatives aux analyses de variance concernant des variables dynamiques (le niveau de signification, la valeur de l'eta, les moyennes et les écarts types pour les pédophiles traités) et aux trois catégories de la variable statut du traitement. Pour les variables dynamiques, il n'y a aucune différence statistiquement significative entre les résultats des sujets des trois catégories de la variable statut du traitement. Cependant, il est à noter que la puissance statistique de ces analyses est faible en raison du nombre restreint de sujets dans chaque groupe. Ainsi, on peut noter que certaines différences entre les moyennes semblent importantes. Plus spécifiquement, l'examen des moyennes des trois catégories de statut du traitement (voir tableau 5) indique que les sujets qui ont reçu un traitement prolongé ont présenté des indices moyens de pédophilie homosexuelle ainsi qu'un niveau moyen d'adhésion à des croyances pédophiles supérieur à ceux des deux autres groupes. Cependant, ces pédophiles ont manifesté moins de résultats moyens supérieurs à 74 , ou à 84 , aux échelles du MCMI, que les sujets des deux autres groupes. 


\section{Tableau 5}

Statistiques relatives aux analyses de variance concernant les variables dynamiques chez des pédophiles selon le statut du traitement

\begin{tabular}{|c|c|c|c|c|c|c|}
\hline Variable & $p$ & Eta & $\mathbf{N}$ & $\begin{array}{l}\text { Moyenne (écart } \\
\text { type) pour le } \\
\text { traitement non } \\
\text { complété }\end{array}$ & $\begin{array}{c}\text { Moyenne (écart } \\
\text { type) pour le } \\
\text { traitement } \\
\text { complété }\end{array}$ & $\begin{array}{c}\text { Moyenne (écart } \\
\text { type) pour le } \\
\text { traitement } \\
\text { prolongé }\end{array}$ \\
\hline $\begin{array}{l}\text { IP hét. attouche- } \\
\text { ment non résistant }\end{array}$ &, 41 &, 20 & 45 & $1,57(1,48)$ & $1,26(1,54)$ & $2,07(1,99)$ \\
\hline $\begin{array}{l}\text { IP hét. attouche- } \\
\text { ment résistant }\end{array}$ &, 58 &, 16 & 47 & $1.42(1,09)$ & $1,08(, 69)$ & $1,39(1,16)$ \\
\hline IP hét. pénétration &, 58 &, 15 & 48 & $1,76(1,82)$ & $1,18(1,72)$ & $1,84(2,65)$ \\
\hline $\begin{array}{l}\text { IP hom. attouche- } \\
\text { ment non résistant }\end{array}$ &, 40 &, 19 & 53 & $2,24(2,62)$ & $2,15(2,88)$ & $3,79(5,83)$ \\
\hline $\begin{array}{l}\text { IP hom. attouche- } \\
\text { ment résistant }\end{array}$ &, 31 &, 22 & 50 & $1,39(1,39)$ & $1,14(, 79)$ & $2,48(4,43)$ \\
\hline IP hom. pénétration &, 97 &, 03 & 49 & $1,38(1,66)$ & $1,53(2,10)$ & $1,45(1,50)$ \\
\hline IP global &, 62 & .13 & 59 & $1,60(1,13)$ & $1,94(2,12)$ & $2,31 \quad(3,04)$ \\
\hline $\begin{array}{l}\text { Nombre d'échelles } \\
\text { du MCMI > } 74\end{array}$ &, 08 &, 53 & 18 & $4,50(2,38)$ & $4,78(1,39)$ & $2,40(1,95)$ \\
\hline $\begin{array}{l}\text { Nombre d'échelles } \\
\text { du MCMI > } 84\end{array}$ &, 14 &, 48 & 18 & $3,00(1,41)$ & $2,67(1,22)$ & $1,40(1,14)$ \\
\hline Anxiété-état & .51 & .21 & 32 & $51,51(11,18)$ & $44,79(12,61)$ & $49,19(19.55)$ \\
\hline Anxjété-trait. & .61 & .18 & 31 & $52,00(6,81)$ & $48,10(9,34)$ & $48,64(13,91)$ \\
\hline Dépression &, 56 &, 20 & 30 & $24,67(9,33)$ & $23,86(14,76)$ & $18.14(13,31)$ \\
\hline Intimité & .46 &, 22 & 33 & $120,29(25,14)$ & $131,87(23,09)$ & $123,69(19,14)$ \\
\hline Satisfaction de vie &, 62 & .18 & 29 & $15,7 \quad(6,36)$ & $16,43(8,86)$ & $13,12(6,93)$ \\
\hline $\begin{array}{l}\text { Croyances } \\
\text { pédophiles }\end{array}$ & .14 &, 37 & 30 & $103,22(16,83)$ & $108,92(16,18)$ & $118,00(12,41)$ \\
\hline Colère &, 94 &, 06 & 32 & $284,10(50,10)$ & $277,14(54,11)$ & $280,62(44,40)$ \\
\hline $\begin{array}{l}\text { Signification }(p) \\
\text { Hétérosexuel (hét) }\end{array}$ & \multicolumn{6}{|c|}{$\begin{array}{l}\text { Nombre de sujets (N) } \\
\text { Homosexuel (hom) } \\
\text { ultiaxial de Millon (MCMI) }\end{array}$} \\
\hline
\end{tabular}

\section{Violeurs}

Au tableau 6, on trouve les taux de récidive (sexuelle, violente, contre les biens, contre les personnes, totale) pour des violeurs dont le traitement est soit non complété, soit complété, soit prolongé. Puisqu'il n'y a pas de lien significatif entre le type de milieu thérapeutique (interne, externe) et le statut du traitement, seules les analyses portant sur les données regroupées des deux types de traitement seront présentées. Ainsi, il n'y a pas de lien 
significatif entre le statut du traitement et les taux de récidive sexuelle, $\chi^{2}(2)=1,3, p=, 5$, phi $=, 14$, violente, $\chi^{2}(2)=2,8, p=, 2$, phi $=, 2$, contre les biens, $\chi^{2}(2)=3,8, p=, 15$, phi $=, 2$, et contre les personnes, $\chi^{2}(2)=4,3$, $p=, 1$, phi $=, 25$. Cependant, il y a un lien significatif entre le statut du traitement et le taux de récidive totale, $\chi^{2}(2)=6,1, p<, 05$, phi $=, 29$.

Tableau 6

Taux (fréquence) de réciđive chez des violeurs selon le statut du traitement

\begin{tabular}{|l|c|c|c|c|c|c|}
\hline & \multicolumn{5}{|c|}{ TYPE DE RÉCIDIVE } \\
\hline Traitement & N & Sexuelle & Violente & $\begin{array}{l}\text { Contre les } \\
\text { biens }\end{array}$ & $\begin{array}{l}\text { Contre les } \\
\text { personnes }\end{array}$ & Totale \\
\hline $\begin{array}{l}\text { Non complété } \\
\text { (moins de 12 mois) }\end{array}$ & 24 & $20,8 \%(5)$ & $50 \%(12)$ & $37,5 \%(9)$ & $54,2 \%(13)$ & $70,8 \%(17)$ \\
\hline $\begin{array}{l}\text { Complété } \\
\text { (de 12 à 24 mois) }\end{array}$ & 20 & $35 \%(7)$ & $40 \%(8)$ & $55 \%(11)$ & $50,2 \%(10)$ & $65 \%(13)$ \\
\hline $\begin{array}{l}\text { Prolongé } \\
\text { (plus de 24 mois) }\end{array}$ & 26 & $23,1 \%(6)$ & $26,9 \%(7)$ & $26,9 \%(7)$ & $26,9 \%(7)$ & $38,5 \%(10)$ \\
\hline
\end{tabular}

Des analyses de régression logistique furent également effectuées, afin de vérifier s'il existe des liens significatifs entre les taux de récidive et le statut du traitement chez les violeurs. Lors de ces analyses, il fut contrôlé pour l'impact de variables qui affectent les taux de récidive : l'âge du sujet, ses antécédents judiciaires sexuels, violents et contre les biens, la durée de la période de suivi et, finalement, le type de milieu thérapeutique (Proulx et coll., 1995). Tout comme dans le cas des pédophiles, nous n'avons pas contrôlé les variables dynamiques parce que le nombre de sujets pour lesquels les données étaient disponibles était insuffisant. Ainsi, lors d'une première étape, les variables qui affectent les taux de récidive furent introduites dans les analyses de régression logistique. Puis, dans une seconde étape, la variable statut du traitement fut introduite dans les analyses de régression logistique. La nature catégorielle de la variable statut du traitement a conduit à la création de deux vecteurs dichotomiques qui permettent d'utiliser la catégorie "traitement complété », comme la catégorie de référence à laquelle sont comparées les catégories «traitement non complété " et «traitement prolongé " lors de la seconde étape des analyses de régression logistique.

Au tableau 7 , on retrouve dans les cinq types de récidive chez des violeurs traités des statistiques décrivant la performance des modèles soumis aux équations de régression logistique : l'amélioration du chi-carré, le nombre de degrés de liberté, le niveau de signification du $\chi^{2}$. Pour les 
cinq types de récidive, l'amélioration du chi-carré pour la variable statut du traitement est non significative.

\section{Tableau 7}

Statistiques relatives aux analyses de régression logistique pour cinq types de récidive chez des violeurs traités $(\mathrm{N}=70)$

\begin{tabular}{|c|c|c|c|c|}
\hline Type de récidive & $\begin{array}{c}\text { Étape de } \\
\text { l'analyse de } \\
\text { régression } \\
\text { logistique }\end{array}$ & $\chi^{2}$ & $\mathbf{D L}$ & $p$ \\
\hline Sexuelle & $\begin{array}{l}\text { Étape } 1 \\
\text { Étape } 2\end{array}$ & $\begin{array}{c}16,13 \\
1,5\end{array}$ & $\begin{array}{l}5 \\
2 \\
\end{array}$ & $\begin{array}{l}.007 \\
, 47\end{array}$ \\
\hline Violente & $\begin{array}{l}\text { Étape } 1 \\
\text { Étape } 2\end{array}$ & $\begin{array}{r}20,09 \\
4,04\end{array}$ & $\begin{array}{l}5 \\
2\end{array}$ & $\begin{array}{l}, 001 \\
, 13\end{array}$ \\
\hline Contre les biens & $\begin{array}{l}\text { Étape } 1 \\
\text { Étape } 2\end{array}$ & $\begin{array}{r}22,19 \\
5,13\end{array}$ & $\begin{array}{l}5 \\
2\end{array}$ & $\begin{array}{l}, 001 \\
, 08\end{array}$ \\
\hline Contre les personnes & $\begin{array}{l}\text { Étape } 1 \\
\text { Étape } 2\end{array}$ & $\begin{array}{r}34,87 \\
2,13\end{array}$ & $\begin{array}{l}5 \\
2\end{array}$ & $\begin{array}{l}, 001 \\
, 35\end{array}$ \\
\hline Totale & $\begin{array}{l}\text { Étape } 1 \\
\text { Étape } 2\end{array}$ & $\begin{array}{r}38,08 \\
2,26\end{array}$ & $\begin{array}{l}5 \\
2\end{array}$ & $\begin{array}{l}, 001 \\
, 33\end{array}$ \\
\hline \multicolumn{5}{|c|}{$\begin{array}{l}\text { Nombre de sujets }(\mathrm{N}) \text {. } \\
\text { Amélioration du chi carré }\left(\chi^{2}\right) \text {. } \\
\text { Nombre de degré de liberté (DL). } \\
\text { Signification }(p) \text {. } \\
\text { Étape } 1: \text { Statistiques de l'équation avec les variables contrôle qui exercent un } \\
\text { effet sur le taux de récidive. } \\
\text { Étape } 2 \text { : Statistiques de l'équation avec la variable traitement, après soustraction } \\
\text { de l'effet des variables contrôle (Improvement : amélioration de l'ajustement). }\end{array}$} \\
\hline
\end{tabular}

Au tableau 8, on trouve des statistiques relatives aux analyses de variance concernant des variables dynamiques: le niveau de signification, la valeur de l'eta, les moyennes et les écarts types pour les violeurs traités aux trois catégories de la variable statut du traitement. Dans le cas de ces variables dynamiques, il n'y a aucune différence statistiquement significative entre les résultats des sujets des trois catégories de la variable statut du traitement. Cependant, l'examen des moyennes de ces trois catégories de statut du traitement (voir tableau 8) indique que les sujets qui n'ont pas complété le traitement présentent des moyennes d'anxiété-état, de niveau de croyances erronées favorisant le viol et de propension à la colère plus élevées que les sujets des deux autres groupes. 
Tableau 8

Statistiques relatives aux analyses de variance concernant les variables dynamiques chez des violeurs selon le statut du traitement

\begin{tabular}{|c|c|c|c|c|c|c|}
\hline Variable & $p$ & Eta & $\mathbf{N}$ & $\begin{array}{l}\text { Moyenne (ecart } \\
\text { type) pour le } \\
\text { traitement non } \\
\text { complété }\end{array}$ & $\begin{array}{c}\text { Moyenne (écart } \\
\text { type) pour le } \\
\text { traitement } \\
\text { complété }\end{array}$ & $\begin{array}{c}\text { Moyenne (écart } \\
\text { type) pour le } \\
\text { traitement } \\
\text { prolongé }\end{array}$ \\
\hline $\begin{array}{l}\text { Indice de viol } \\
\text { physique }\end{array}$ &, 96 &, 05 & 37 & $1,3(1,22)$ & $1,2(, 66)$ & $1,2(, 85)$ \\
\hline Indice d'agression & .66 &, 16 & 36 & $6(, 55)$ &, $4(, 26)$ & $.5(, 56)$ \\
\hline $\begin{array}{l}\text { Nombre } \\
\text { d'échelles du } \\
\text { MCMI>74 }\end{array}$ &, 97 & .07 & 14 & $4,0(1,82)$ & $4,2(1,71)$ & $4,3(2,80)$ \\
\hline $\begin{array}{l}\text { Nombre } \\
\text { d'échelles du } \\
\text { MCM1.84 }\end{array}$ &, 88 &, 15 & 14 & $2,5 \quad(1,91)$ & $3,0(2,16)$ & $2,3(2,25)$ \\
\hline Anxiétế-état &, 05 &, 07 & 20 & $49,3(22,75)$ & $46,2(14,67)$ & $47,0(11,88)$ \\
\hline Anxiété-trait &, 60 &, 24 & 20 & $54,3(23,54)$ & $46,2(7.26)$ & $50,0(8,13)$ \\
\hline Dépression & .80 &, 16 & 20 & $13,3(13,32)$ & $14,6(9,69)$ & $17,1 \quad(9,51)$ \\
\hline Intimité &, 98 &, 05 & 17 & $132,3(44,87)$ & $131,4(19,14)$ & $134,0(17,43)$ \\
\hline Satisfaction de vie & .66 &, 22 & 18 & $20,5(17,7)$ & $16,0(6,2)$ & $15,2(7,5)$ \\
\hline $\begin{array}{l}\text { Croyances sur le } \\
\text { viol }\end{array}$ &, 14 &, 48 & 18 & $109,5(4,10)$ & $94,2(17,48)$ & $90,3(16,62)$ \\
\hline Colère & .67 &, 21 & 20 & $302,0(80,22)$ & $291,4(51,02)$ & $274,6(47,10)$ \\
\hline \multicolumn{7}{|c|}{$\begin{array}{l}\text { Signification }(p) . \quad \text { Nombre de sujets }(\mathrm{N}) . \quad \text { Moyenne }(\overline{\mathrm{X}}) \text {. } \\
\text { Inventaire clinique multiaxial de Millon }(\mathrm{MCMI}) .\end{array}$} \\
\hline
\end{tabular}

\section{INTERPRÉTATION DES RÉSULTATS}

En ce qui a trait aux pédophiles, nos résultats indiquent que ceux quj n'ont pas complété le programme de traitement présentent des taux de récidive sexuelle et violente plus êlevés que ceux qui l'ont complété (voir tableau 2). Toutefois, les analyses univariées et multivariées effectuées indiquent que ces différences ne sont pas statistiquement significatives. Les résultats de l'étude de Marques et coll. (1994) démontrent également que les taux de récidive sexuelle et violente des agresseurs sexuels (7 pédophiles, 1 violeur) n'ayant pas complété un programme de traitement sont plus élevés que ceux des agresseurs sexuels l'ayant complété. Néanmoins, seule la différence quant aux taux de récidive sexuelle est statistiquement significative. Bien que nos résultats et ceux de l'étude de Marques et coll. (1994) convergent, les résultats des analyses statistiques effectuées diffèrent partiellement. Il est possible que ces différences soient attribuables au fait 
que nous n'avons pas utilisé les mêmes analyses statistiques. En effet, nous avons eu recours à des analyses de régression logistique, alors que Marques et coll. ont effectué des analyses de survie paramétriques. De plus, il se peut que les résultats de nos analyses statistiques diffèrent de ceux de Marques et coll. en raison de la composition des groupes comparés. De fait, dans nos analyses, les deux groupes comparés ne comprenaient que des pédophiles, alors que dans celles de Marques et coll. les deux groupes comprenaient des violeurs et des pédophiles. Il est également intéressant de souligner qu'il y a peu de différences statistiquement significatives entre les divers groupes dans notre étude comme dans celle de Marques et coll. (1994). Une telle situation peut être attribuable à la faible puissance statistique (Cohen, 1992) généralement observée dans les études portant sur l'efficacité des programmes de traitement plutôt qu'à l'absence d'impact de ces programmes (Weisburd, Petrosino et Mason, 1993).

Chez les pédophiles dont le traitement est complété, le taux de récidive contre les personnes ne diffère pas significativement de celui des pédophiles dont le traitement est non complété. Un tel résultat n'est pas surprenant car, chez les pédophiles de notre étude, la récidive contre les personnes consiste principalement en des récidives sexuelles pour lesquelles il n'y a pas de différence statistiquement significative. À un autre niveau, le taux de récidive contre les biens et le taux de récidive totale sont significativement plus élevés chez les pédophiles n'ayant pas complété le programme de traitement que chez ceux l'ayant complété. De tels résultats donnent l'impression qu'un programme élaboré afin de réduire le taux de récidive sexuelle chez des agresseurs sexuels a un impact non pas à ce niveau, mais plutôt sur le taux de récidive contre les biens et sur le taux de récidive totale. Une telle impression est trompeuse, car le taux de récidive des pédophiles dont le traitement est non complété est plus élevé pour les crimes contre les biens que pour les crimes sexuels. Ainsi, même si le fait de compléter le programme de traitement réduisait à $0 \%$ les taux de récidive sexuelle et les taux de récidive contre les biens, il est possible que cette réduction ne soit statistiquement significative que pour les crimes contre les biens, et ce parce que le taux de base de récidive officielle pour ce type de crime permet une plus grande réduction du taux de récidive que pour les crimes sexuels. Le problème des faibles taux de base de récidive sexuelle officielle a déjà été invoqué par Marshall et Pithers (1994) afin d'expliquer la difficulté à démontrer l'efficacitê des programmes de traitement pour réduire les taux de récidive chez les agresseurs sexuels.

Une solution invoquée afin d'augmenter les taux de base de récidive officielle consiste à considérer une période de suivi plus longue (Furby et coll., 1989; Hanson et coll., 1993; Quinsey, 1980). Cependant, même si l'on règle le problème des taux de base, il reste à expliquer pourquoi un programme destiné à réduire le taux de récidive sexuelle réduit également 
le taux de récidive contre les biens. Une hypothèse serait que les pédophiles, en cours de traitement, acquièrent des habiletés leur permettant d'être mieux adaptés socialement et professionnellement, ce qui rendrait moins nécessaire l'utilisation de moyens antisociaux pour satisfaire leurs besoins matériels.

Le taux de récidive chez les pédophiles ayant rę̧u un traitement prolongé est plus élevé que chez ceux ayant complété le traitement, et ce pour les cinq formes de récidive examinées. Toutefois, ces différences ne sont statistiquement significatives que pour les récidives contre les personnes et totale. Une hypothèse permettant d'expliquer ces résultats contre-intuitifs est que les pédophiles ayant reçu un traitement prolongé étaient les cas les plus pathologiques et que cette situation aurait justifié l'application de mesures thérapeutiques exceptionnelles.

Afin de vérifier cette hypothèse, nous avons comparé les pédophiles ayant reçu un traitement prolongé à ceux ayant complété le traitement au niveau de leurs préférences sexuelles et des caractéristiques de leur personnalité. Les analyses effectuées ne comportent aucune différence statistiquement significative entre les deux groupes. Toutefois, on peut souligner que les pédophiles ayant reçu un traitement prolongé présentent des préférences sexuelles plus déviantes et des croyances pédophiles plus prononcées que les pédophiles ayant complété le traitement. Ainsi, si l'on tente de dresser un portrait des pédophiles ayant reçu un traitement prolongé, il semble que ceux-ci soient fortement enracinés dans la pédophilie. Ces résultats contrastent avec ceux d'Abel et coll. (1988), lesquels indiquent que les pédophiles n'ayant pas complété le traitement présentent plusieurs paraphilies.

En ce qui a trait aux violeurs, nos résultats indiquent que ceux qui ont reçu un traitement prolongé présentent des taux de récidive officielle violente, contre les biens, contre les personnes et totale inférieurs à ceux des deux autres groupes. Les analyses statistiques univariées ne confirment que la différence observée pour la récidive totale, alors que les analyses multivariées ne comportent aucune différence statistiquement significative. Comme dans le cas des pédophiles, il est possible qu'une telle situation soit attribuable à la faible puissance statistique généralement observée dans les études portant sur l'efficacité des programmes de traitement plutôt qu'à l'absence d'impact de ces programmes. Puisque dans l'étude de Marques et coll. (1994) il n'y a qu'un seul violeur n'ayant pas complété le traitement et qu'il n'y a pas de groupe de violeurs ayant reçu un traitement prolongé, il est impossible de comparer les résultats de cette étude à ceux obtenus auprès des violeurs de notre étude.

Des analyses statistiques furent effectuées afin de vérifier si les résultats obtenus pour les facteurs dynamiques, chez les violeurs, diffèrent selon le statut du traitement. Aucune de ces analyses ne comporte de différence 
statistiquement significative entre les trois groupes. Toutefois, on peut souligner que les violeurs n'ayant pas complété le traitement présentent des croyances favorisant le viol et une propension à la colère plus prononcées que les violeurs des deux autres groupes. Ces deux facteurs concordent avec une fin prématurée du programme de traitement. En effet, ces violeurs seraient plus susceptibles d'être en conflit avec les membres de l'équipe traitante et ils seraient moins susceptibles de reconnaître le caractère déviant de leurs actes délictueux. En outre, l'absence de différence, quant aux facteurs dynamiques, entre les violeurs ayant complété le traitement et ceux ayant reçu un traitement prolongé favoriserait l'hypothèse selon laquelle des taux de récidive plus bas chez ces derniers seraient attribuables à un impact positif du traitement.

Les résultats de notre étude indiquent que le traitement a un impact différent chez les pédophiles et les violeurs. En effet, dans le cas des pédophiles ayant complété le traitement, les divers taux de récidive officielle sont inférieurs à ceux des pédophiles n'ayant pas complété le traitement. Toutefois, les pédophiles ayant reçu un traitement prolongé présentent des taux de récidive plus élevés que ceux ayant complété le traitement. Nous avons formulé l'hypothèse que ces demiers résultats seraient attribuables à un enracinement plus prononcé dans la déviance sexuelle chez les pédophiles ayant reçu un traitement prolongé. Ainsi, afin de réduire le taux de récidive chez ce type particulier de pédophiles, il serait nécessaire d'adapter les modalités de traitement et d'encadrement qui leur sont destinés (ex. : castration chimique, suivi post-traitement intensif). En ce qui a trait aux violeurs, le taux de récidive sexuelle chez ceux ayant reçu un traitement prolongé n'est pas inférieur aux taux des deux autres groupes. Cependant, les taux de récidive (violente, contre les biens, contre les personnes, totale) chez ceux ayant reçu un traitement prolongé (plus de 24 mois) sont plus bas que les taux observés dans les deux autres groupes. Ainsi, il semble que, avec les violeurs, le programme de traitement de l'Institut Philippe-Pinel de Montréal exerce un impact positif lorsque sa durée est supérieure à 24 mois. Toutefois, des ajustements devraient être apportés au programme actuel, afin d'accrôtre son impact au niveau de la récidive sexuelle chez les violeurs.

Notre étude est la première au cours de laquelle l'impact de l'abandon précoce d'un programme de traitement sur les taux de récidive officielle fut évalué séparément chez des violeurs et des pédophiles. De plus, elle est la seule au cours de laquelle l'impact d'un traitement prolongé sur les taux de récidive officielle fut examiné chez des agresseurs sexuels. Toutefois, en raison de contraintes éthiques et administratives, notre étude ne comporte pas de groupes témoins constitués d'agresseurs sexuels non traités. Ainsi, l'évaluation de l'efficacité du programme de traitement de l'Institut Philippe-Pinel de Montréal demeure incomplète. De plus, l'intérêt des 
analyses portant sur l'impact des facteurs dynamiques sur le statut du traitement est limité, et ce parce que les données relatives à ces facteurs n'étaient disponibles que pour un nombre restreint de sujets. En conséquence, des études supplémentaires devront être réalisées afin de clarifier l'impact des facteurs dynamiques sur le statut du traitement.

\section{BIBLIOGRAPHIE}

ABEL, G. G., BLANCHARD, E. B., BECKER, J. V. et DJENDEREDJIAN, A. (1978), "Differentiating sexual aggressives with penile measures", Criminal Justice and Behavior, vol. 5, pp. 315-332.

ABEL, G. G., GORE, D. K., HOLLAND, C. L., CAMP, N., BECKER, J. V. et RATHNER, J. ( (989), "Cognitive distortions of child molesters », Annals of Sex Research, vol. 2, pp. 135-153.

ABEL, G. G., MITTELMAN, M. S., BECKER, J. V., RATHNER, J. et ROULEAU, J. L. (1988), «Predicting child molesters' response to treatment», in R. A. PRENTKY et V. L. QUINSEY (dir.), Human Sexual Aggression : Current perspectives, New York, Annals of the New York Academy of Science, pp. 223-234.

AUBUT, J. (1993), Les Agresseurs sexuels : théorie, évaluation et traitement. Montréal, Les Éditions de la Chenelière.

BANCROFT, J., JONES, H. G. et PULLAN, B. R. (1966), "A simple transducer for measuring penile erection with comments on its use in the treatment of sexual disorders ", Behaviour Research and Therapy, vol. 4, pp. 239-241.

BECK, A. T., WARD, C. H., MENDELSON, M., MOCK, J. et ERBAUGH, J. (1961), "An inventory for measuring depression", Archives of General Psychiatry, vol. 14, pp. 561-571.

BECKER, J. V. et HUNTER, J. A. (1992), « Evaluation of treatment outcome for adult perpetrators of child sexual abuse», Criminal Justice and Behavior, vol. 19, pp. 74-92.

BURT, M. R. (1980), "Cultural myths and support for rape », Journal of Personality and Social Psychology, vol. 38, pp. 217-230.

COHEN, J. (1992), «A power primer», Psychological Bulletin, vol. 112, n 1, pp. 155159.

DAVIDSON, P. (1984), Outcome data for a penitentiary-based treatment program for sex offenders, communication présenté à la Conference on the Assessment and Treatment of the Sex Offender, Kingston, Ontario.

DIX, G. E. (1976), "Determining the continued dangerousness of psychologically abnormal sex offenders », Journal of Psychiatry and the Law, vol. 3, pp. 327-344.

EARLS, C. M. et PROULX, J. (1986), «The differentiation of francophone rapists and non rapists using penile circumferential measures $"$, Criminal Justice and Behavior, vol. 13, pp. 419-429.

FLORIDA DEPARTMENT OF HEALTH AND REHABILITATIVE SERVICES (1976), Evaluation of sex offender rehabilitation programs in the state of Florida, Tampa, Mental Health Program Office. 
FLORIDA DEPARTMENT OF HEALTH AND REHABILITATIVE SERVICES (1984), Status of the sex offender treatment programs, fiscal year 1983-84, Annual Report to the Florida Legislature, Alcohol. Drug Abuse and Mental Health Program Office, Tampa.

FRISBIE, L. V. (1969), Another look at sex offenders in California, Mental Health Research Monograph $N^{\circ} 12$, Sacramento, CA, Californie, Department of Mental Hygiene.

FURBY, L., WEINROTT, M. R. et BLACKSHAW, L. (1989), «Sex offender recidivism ", Psychological Bulletin, vol. 105, pp. 3-30.

GAGNÉ De, R. (1987). Distinction entre des agresseurs sexuels d'enfants et des non agresseurs sexuels d'enfants à l'aide de stimuli sonores, mémoire de maitrise inédit, Département de psychologie, Université de Montréal.

HANSON, R. K., STEFFY, R. A. et GAUTHIER, R. (1993), « Long-term recidivism of child molesters ", Journal of Consulting and Clinical Psychology, vol. $61, \mathrm{n}^{\circ} 4$, pp.646-652.

KELLY, R. J. (1982), "Behavioral reorientation of pedophiliacs : Can it be done? ", Clinical Psychology Review, vol. 2, pp. 387-408.

MARQUES, J. K., DAY, D. M., NELSON, C. et WEST, M. A. (1994), «Effects of cognitive-behavioral treatment on sex offender recidivism : Preliminary results of a longitudinal study ", Criminal Justice and Behavior, vol. 21, pp. 28-54.

MARSHALL, W. L. et BARBAREE, H. E. (1988), "The long-term evaluation of a behavioral treatment program for child molesters ", Behaviour Research and Therapy, vol. 26, pp. 499-511.

MARSHALL, W. L., JONES, R., WARD, T., JOHNSTON, P. et BARBAREE, H. E. (1991), "Treatment outcome with sex offenders", Clinical Psychology Review, vol. 11, pp. 465-485.

MARSHALL, W. L. et PITHERS, W. L. (1994), "A reconsideration of treatment outcome with sex offenders », Criminal Justice and Behavior, vol. 21, pp. 10-27.

McKIBBEN, A. et PROULX, J. (1991), «Évaluation et traitement des agresseurs sexuels à l'Institut Philippe-Pinel de Montréal ", Bulletin de l'Association des sexologues du Québec, vol. 12, pp. 17-26.

MEYERS, L. C. et ROMERO, J. J. (1980), A ten-year follow-up of sex offender recidivism, Philadelphie, J. J. Peters Institute.

MILLER, R. S. et LEFCOURT, H. M. (1982), «The assessment of intimacy », Journal of Personality Assessment, vol. 66, pp. 514-518.

MILLON, T. (1983), Millon Clinical Multiaxial Inventory Manual, Minneapolis, Interpretative Scoring Systems.

NOVACO, R. W. (1975), Anger Control, Lexington, Lexington Press.

PAVOT, W. (1993), «Review of the satisfaction with life scale", Psychological Assessment, vol. 5, pp. 164-172.

PETERS, J. J. et ROETHER, H. A. (1971), Success and Failure of Sex Offenders, Philadelphie, American Association For the Advancement of Science.

PROULX, J. (1989), "Sexual preference assessment of sexual aggressors ", International Journal of Law and Psychiatry, vol. 12, pp. 275-280. 
PROULX, J., AUBUT, J., McKIBBEN, A. et CÔTÉ, M. (1994), «Penile responses of rapists and non rapists to rape stimuli involving violence or humiliation", Archives of Sexual Behavior, vol. 23, pp. 295-310.

PROULX, J., PELLERIN, B., PARADIS, Y., McKIBBEN, A., AUBUT, J. et OUIMET, M. (1995), "Static and dynamic predictors of recidivism in sexual aggressors". Soumis pour publication.

QUINSEY, V. L. (1980), «The baserate problem and the prediction of dangerousness : A reappraisal ", Journal of Psychiatry and Law, vol. 8, pp. 329-340.

QUINSEY, V. L. et CHAPLIN, T. C. (1988), « Penile responses of child molesters and normals to descriptions of encounters with children involving sex and violence ", Journal of Interpersonal Violence, vol. 3, 259-274.

QUINSEY, V. L., HARRIS, G. T., RICE, M. E. et LALUMIÈRE, M. L. (1993), «Assessing treatment efficacity in outcome studies of sex offenders », Journal of Interpersonal Violence, vol. $8, n^{\circ} 4$, pp. 512-523.

QUINSEY, V. L. et LAWS, D. R. (1990), «Validity of physiological measures of pedophilic sexual arousal in a sexual offender population : A critique of Hall, Proctor et Nelson », Journal of Consulting and Clinical Psychology, vol. 58, pp. 886-888.

RICE, M. E., HARRIS, G. T. et QUINSEY, V. L. (1993), « Evaluating treatment programs for child molesters ", in J. HUDSON et I. V. ROBERTS (dir.), Evaluation Research in Canadian Justice Programs, Calgary, University of Calgary Press.

RICE, M. E., QUINSEY, V. L. et HARRIS, G. T. (1991), "Sexual recidivism among child molesters released from a maximum security psychiatric institution ", Journal of Consulting and Clinical Psychology, vol. 59, pp. 381-386.

ROMERO, J. J. et WILLIAMS, L. M. (1985), « Recidivism among convicted sex offenders : A ten-year follow-up study ", Federal Probation, vol. 49, pp. 58-64.

SPIELBERGER, C. G., GORSUCH, R. L. et LUSHENE, R. D. (1970), Manual for the State-Trait Anxiety Inventory (Self-Evaluation Questionnaire), Palo Alto, Consulting Psychologist Press.

STURGEON, V. H. et TAYLOR, J. (1980), « Report of a five-year follow-up study of mentally disordered sex offenders released from Atascadero State Hospital in 1973 », Criminal Justice Journal, vol. 4, n० 31, pp. 31-63.

WEISBURD, D., PETROSINO, A. et MASON, G. (1993), "Design sensitivity in criminal justice experiments », M. Tonry (dir.), in Crime and Justice : A Review of Research, Chicago, The Chicago University Press, vol. 17, pp. 337-378.

\section{REMERCIEMENTS}

La réalisation de cette étude fut possible grâce à une subvention du Solliciteur général du Canada (9514-IN/525). Toutefois, les opinions émises dans cet article n'engagent que leurs auteurs.

Nous tenons à remercier les personnes suivantes pour leur apport à la réalisation de cette étude : Julie Garceau, Carole Hébert, Bernadette Lamoureux, Nicole Perron, Monique Tardif et Rose May Nahabet.

Toute correspondance concernant cet article doit être adressée à Jean Proulx, Ph. D., Université de Montréal (Centre international de criminologie comparée, École de criminologie), C.P. 6128, Succ. Centre-ville, Montréal, Québec, Canada, H3C 3J7. 